\title{
Perfect locales and localic real functions
}

\author{
Javier Gutiérrez García*, Tomasz Kubiak and Jorge Picado
}

\begin{abstract}
The purpose of this paper is to identify the role of perfectness in the Michael insertion theorem for perfectly normal locales. We attain it by characterizing perfect locales in terms of strict insertion of two comparable lower semicontinuous and upper semicontinuous localic real functions. That characterization, when combined with the insertion theorem for normal locales, provides an improved formulation of the aforementioned pointfree form of Michael's insertion theorem.
\end{abstract}

Mathematics Subject Classification. 06D22, 18F70, 54D15.

Keywords. Locale, Sublocale, Perfectness, $G_{\delta}$-perfectness, Perfect normality, Semicontinuous real function, Insertion theorem.

\section{Introduction}

Recall that a topological space is perfect if each open set is $F_{\sigma}$. This is equivalent to the statement that each closed set is $G_{\delta}$. In the category of locales, these two formulations are no longer equivalent when phrased in terms of open sublocales and closed sublocales, in which case $G_{\delta}$-perfectness is generally stronger than $F_{\sigma}$-perfectness. In [7], we took the weaker condition as the pointfree concept of perfectness and kept the terminology of a $G_{\delta}$-perfect locale for the locales that satisfy the stronger condition.

In the present paper, we prove that the strict insertion theorem for realvalued functions on perfect topological spaces (as in [4] and [17]) extends to both classes of perfect and $G_{\delta}$-perfect locales, with a slightly different formulation in the former case. Indeed, it is only under the stronger condition of $G_{\delta}$-perfectness that the strict double insertion scheme can be formulated as in the classical case. We then revisit our pointfree version of Michael's strict

\footnotetext{
${ }^{*}$ Corresponding author.

The authors acknowledge financial support from the Ministry of Economy and Competitiveness of Spain (grant MTM2015-63608-P (MINECO/FEDER, UE)), from the Basque Government (grant IT974-16) and from the Centre for Mathematics of the University of Coimbra (grant UID/MAT/00324/2019, funded by the Portuguese Government through FCT/MEC and ERDF Partnership Agreement PT2020).
} 
insertion theorem for perfectly normal spaces in [5] and present an improved version of it.

\section{Preliminaries}

We present in this section a brief survey of the background required. A classic reference to locales and frames is Johnstone [11]. Our main reference in this paper is Picado-Pultr [15] for which we refer for all the terms not defined herein.

\subsection{Locales and sublocales}

A locale (or frame) is a complete lattice $L$ in which

$$
a \wedge \bigvee B=\bigvee\{a \wedge b \mid b \in B\}
$$

for all $a \in L$ and $B \subseteq L$. The universal bounds are denoted 0 and 1 (if not stated otherwise). A frame homomorphism is a map $h: L \rightarrow M$ between frames that preserves finite meets and arbitrary joins (hence the universal bounds too).

For any topological space $X$, its topology $\Omega(X)$ is a locale (under inclusion). For any continuous function $f: X \rightarrow Y$, the inverse image map determines a frame homomorphism $\Omega(Y) \rightarrow \Omega(X)$. A locale $L$ isomorphic to some $\Omega(X)$ is called spatial.

Each frame $L$ is a complete Heyting algebra with Heyting operator (implication) given by $a \rightarrow b=\bigvee\{c \in L \mid a \wedge c \leq b\}$. In particular, the pseudocomplement of an $a \in L$ is the element $a^{*}=a \rightarrow 0$. Then $a \wedge a^{*}=0$ and $(\bigvee A)^{*}=\bigwedge_{a \in A} a^{*}$ for all $A \subseteq L$.

A subset $S$ of a locale $L$ is a sublocale if

$$
\bigwedge A \text { and } a \rightarrow s \text { are in } S
$$

for all $A \subseteq S, a \in L$, and $s \in S$. Since an arbitrary intersection of sublocales is again a sublocale, the system

$$
\mathcal{S}(L)
$$

of all sublocales of $L$ is a complete lattice. Meets and joins are given by

$$
\bigwedge_{i \in I} S_{i}=\bigcap_{i \in I} S_{i} \text { and } \bigvee_{i \in I} S_{i}=\left\{\bigwedge A \mid A \subseteq \bigcup_{i \in I} S_{i}\right\}
$$

with universal bounds $0=\{1\}$ and $1=L$. This lattice is a coframe, that is, the dual of a frame (cf. [15, III.3.2.1]). Hence $\mathcal{S}(L)$ is a co-Heyting algebra and each $S$ of $\mathcal{S}(L)$ has a co-pseudocomplement $S^{\#}$ determined by the formula

$$
S^{\#}=\bigcap\{T \in \mathcal{S}(L) \mid S \vee T=L\} .
$$

In particular, $S^{\#}=\{1\}$ iff $S=L$ and $\left(\cap S_{i}\right)^{\#}=\bigvee S_{i}{ }^{\#}$.

For each $a \in L$, the sublocales

$$
\mathfrak{c}(a)=\uparrow a \quad \text { and } \quad \mathfrak{o}(a)=\{a \rightarrow b \mid b \in L\}
$$


are, respectively, the closed and open sublocales induced by $a$. They are complemented to each other in $\mathcal{S}(L)$. The following properties hold for any $a, b \in L$, any $A \subseteq L$ and any finite $F \subseteq L$ :

$$
\begin{aligned}
& \mathfrak{c}(a) \subseteq \mathfrak{o}(b) \quad \text { iff } \quad a \vee b=1, \\
& \bigcap_{a \in A} \mathfrak{c}(a)=\mathfrak{c}(\bigvee A), \quad \bigvee_{a \in A} \mathfrak{o}(a)=\mathfrak{o}(\bigvee A), \text { and } \\
& \bigvee_{a \in F} \mathfrak{c}(a)=\mathfrak{c}(\bigwedge F), \quad \bigcap_{a \in F} \mathfrak{o}(a)=\mathfrak{o}(\bigwedge F) .
\end{aligned}
$$

\subsection{The frame of reals and localic real functions}

Recall the frame of reals $\mathfrak{L}(\mathbb{R})$ from [2]. We shall regard it (see [13] or [15] for more details) as a frame presented by generators and relations, with generators $(r,-)$ and $(-, r)$ for all rational $r$, and relations

(r1) $(r,-) \wedge(-, q)=0$ if $q \leq r$,

(r2) $(r,-) \vee(-, q)=1$ if $r<q$,

(r3) $(r,-)=\bigvee_{q>r}(q,-)$,

(r4) $(-, r)=\bigvee_{q<r}(-, q)$,

(r5) $\bigvee_{r \in \mathbb{Q}}(r,-)=1$,

(r6) $\bigvee_{r \in \mathbb{Q}}(-, r)=1$.

A localic real function on a frame $L$ is a frame homomorphism $\mathfrak{L}(\mathbb{R}) \rightarrow$ $\mathcal{S}(L)^{\mathrm{op}}$. The system

$$
\mathrm{F}(L)
$$

of all them is partially ordered by

$$
f \leq g \quad \text { iff } \quad g(r,-) \subseteq f(r,-) \quad \text { for all } r \in \mathbb{Q} .
$$

Note. Along the paper, we shall always express the computations with sublocales in terms of the coframe $(\mathcal{S}(L), \subseteq$ ). The symbol $\bigvee$ (and $\vee$ ) will be used both for joins in $\mathcal{S}(L)$ and meets in $\mathcal{S}(L)^{\text {op }}$ while $\bigcap$ (and $\cap$ ) will refer to meets in $\mathcal{S}(L)$ and joins in $\mathcal{S}(L)^{\mathrm{o} p}$.

Since each frame homomorphism $f: \mathfrak{L}(\mathbb{R}) \rightarrow \mathcal{S}(L)^{\text {op }}$ is uniquely determined by the images $f(r,-)$ and $f(-, r)$ and turn the relations (r1)-(r6) into identities in $\mathcal{S}(L)^{\mathrm{o} p}$, it satisfies the following properties:

(1) $f(r,-) \vee f(-, q)=L$ whenever $q \leq r$,

(2) $f(r,-) \cap f(-, q)=\{1\}$ whenever $r<q$,

(3) $f(r,-)=\bigcap_{q>r} f(q,-)$,

(4) $f(-, r)=\bigcap_{q<r} f(-, q)$,

(5) $\bigcap_{r \in \mathbb{Q}} f(r,-)=\{1\}$,

(6) $\bigcap_{r \in \mathbb{Q}} f(-, r)=\{1\}$.

A general procedure for constructing such maps $f$ uses scales of sublocales (cf. [6]). Specifically, a family $\left\{S_{r}\right\}_{r \in \mathbb{Q}}$ of sublocales of $L$ forms a scale if

$$
S_{r} \cap S_{q}^{\#}=\{1\} \quad \text { whenever } \quad r<q,
$$


and

Then, the formulas

$$
\bigcap_{r \in \mathbb{Q}} S_{r}=\{1\}=\bigcap_{r \in \mathbb{Q}} S_{r}^{\#}
$$

$$
f(r,-)=\bigcap_{q>r} S_{q} \quad \text { and } \quad f(-, r)=\bigcap_{q<r} S_{q}^{\#}
$$

uniquely determine a frame homomorphism $f: \mathfrak{L}(\mathbb{R}) \rightarrow \mathcal{S}(L)^{\mathrm{o} p}$. We then say that $f$ is the localic real function generated by the scale $\left\{S_{r}\right\}_{r \in \mathbb{Q}}$.

Remark 2.1. By condition (*), a scale is necessarily an isotone family. Conversely, if $\left\{S_{r}\right\}_{r \in \mathbb{Q}}$ is an arbitrary isotone family of sublocales of $L$ and, for each pair $r<q$, there is a complemented sublocale $T$ such that $S_{r} \subseteq T \subseteq S_{q}$, then $\left\{S_{r}\right\}_{r \in \mathbb{Q}}$ satisfies $(*)$ (since $\left.S_{r} \cap S_{q}^{\#} \subseteq T \cap T^{\#}=\{1\}\right)$. In particular, a family $\left\{S_{r}\right\}_{r \in \mathbb{Q}}$ of complemented sublocales satisfies $(*)$ if and only if it is isotone.

A localic real function $f: \mathfrak{L}(\mathbb{R}) \rightarrow \mathcal{S}(L)^{\mathrm{op}}$ is

(1) lower semicontinuous if $f(r,-)$ is a closed sublocale for all $r \in \mathbb{Q}$,

(2) upper semicontinuous if $f(-, r)$ is a closed sublocale for all $r \in \mathbb{Q}$, and

(3) continuous if it is both lower and upper semicontinuous.

Remark 2.2. Members of $\mathrm{F}(\Omega(X))$ are pointfree analogues of arbitrary (not necessarily continuous) real-valued functions on a topological space $X$. Under some weak conditions, semicontinuities of members of $\mathrm{F}(\Omega(X))$ coincide with the standard topological semicontinuities (see [6], [14] and [16] for more information).

\subsection{Some algebraic operations on localic real functions}

It is well known that $\mathrm{F}(L)$ is a lattice-ordered ring. We shall need explicit formulas for addition and multiplication by a positive rational (cf. [9, Section 4]).

For each $f \in \mathrm{F}(L),-f$ is defined by

$$
(-f)(r,-)=f(-,-r) \quad \text { and } \quad(-f)(-, r)=f(-r,-) .
$$

For a given positive rational $p$, let $S_{r}=f\left(\frac{r}{p},-\right)$ for any $r \in \mathbb{Q}$. Then $\left\{S_{r}\right\}_{r \in \mathbb{Q}}$ is a scale, and the localic real function generated by it is denoted as $p f$. In particular, for each $r \in \mathbb{Q}$ we have

$$
(p f)(r,-)=f\left(\frac{r}{p},-\right) \quad \text { and } \quad(p f)(-, r)=f\left(-, \frac{r}{p}\right) .
$$

Furthermore, for any pair $f, g \in \mathrm{F}(L), f+g$ is the function generated by the scale formed by the sublocales

$$
S_{r}=\bigcap_{s \in \mathbb{Q}}(f(s,-) \vee g(r-s,-)) .
$$

In particular, for each $r \in \mathbb{Q}$ we have

$$
(f+g)(r,-)=\bigcap_{s \in \mathbb{Q}}(f(s,-) \vee g(r-s,-))
$$


and

$$
(f+g)(-, r)=\bigcap_{s \in \mathbb{Q}}(f(-, s) \vee g(-, r-s)) .
$$

Let $f-g=f+(-g)$. Note that $f \leq g$ iff $g-f \geq \mathbf{0}$, where the constant function $\mathbf{0} \in \mathrm{F}(L)$ is defined by

$$
\mathbf{0}(r,-)=\left\{\begin{array}{ll}
\{1\} & \text { if } r<0 \\
L & \text { if } r \geq 0
\end{array} \quad \text { and } \quad \mathbf{0}(-, r)= \begin{cases}L & \text { if } r \leq 0 \\
\{1\} & \text { if } r>0\end{cases}\right.
$$

If $f$ and $g$ are lower (resp., upper) semicontinuous, then so is $f+g([9$, Corollary 4.4]).

\section{Measuring strict inequality between localic real functions}

Recall the following order relations on real-valued functions $F, G, H: X \rightarrow \mathbb{R}$ (on a topological space $X$ ):

(O1) $F \leq G$ iff $F(x) \leq G(x)$ for all $x \in X$.

(O2) $F \supsetneqq G$ iff $F \leq G$ and $F \neq G$ (= the strictly less-than relation).

(O3) $F<G$ iff $F(x)<G(x)$ for all $x \in X$.

(O4) $H$ is strictly in-between $F$ and $G$ iff $F \leq H \leq G$ and for any $x \in X$ one has $F(x)<H(x)<G(x)$ whenever $F(x)<G(x)$ (cf. [12]).

A pointfree counterpart of $(\mathrm{O} 1)$, and thus of $(\mathrm{O} 2)$, has been defined in 2.2. Regarding (O3) and (O4), we follow [5, 9] and define a map

$$
\iota: \mathrm{F}(L) \times \mathrm{F}(L) \rightarrow \mathcal{S}(L)^{\mathrm{o} p}
$$

by

$$
\iota(f, g)=\bigcap_{r \in \mathbb{Q}}(f(-, r) \vee g(r,-)) .
$$

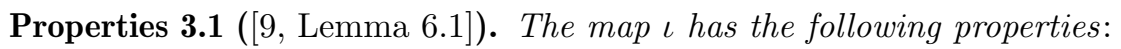

(1) $\iota(\mathbf{0}, f)=f(0,-)$,

(2) $\iota(f, g)=\iota(\mathbf{0}, g-f)$,

(3) $\iota(p f, p g)=\iota(f, g)$ for any rational $p>0$,

(4) If $f_{1} \leq f_{2}$ and $g_{1} \leq g_{2}$, then $\iota\left(f_{1}, g_{2}\right) \subseteq \iota\left(f_{2}, g_{1}\right)$.

Now, for any $f, g \in \mathrm{F}(L)$ we write

$$
f<g \quad \text { whenever } \quad \iota(f, g)^{\#}=L .
$$

Our results below (as well as the insertion theorems of $[5,9]$ ) confirm that this is the right pointfree counterpart, in $\mathrm{F}(L)$, of the order relation (O3).

Remark 3.2. In both [5] and [9] we were always dealing with pairs $(u, l) \in$ $\mathrm{F}(L) \times \mathrm{F}(L)$ where $u$ is upper semicontinuous and $l$ is lower semicontinuous. In that case, $\iota(u, l)$ is a closed sublocale (hence complemented) and, consequently, $\iota(u, l)^{\#}=L$ if and only if $\iota(u, l)=\{1\}$. However, for an arbitrary pair $(f, g)$ in $\mathrm{F}(L) \times \mathrm{F}(L)$ we only have that $\iota(f, g)=\{1\}$ implies $\iota(f, g)^{\#}=L$, but not conversely. 
Lemma 3.3. For any $f, g \in \mathrm{F}(L)$ we have:

(1) $g \leq f \quad$ iff $\quad \iota(f, g)=L \quad$ iff $\quad \iota(f, g)^{\#}=\{1\}$.

(2) $f<g$ implies $f \supsetneqq g$.

(3) $f<g$ and $g<h$ imply $f<h$.

Proof. (1) If $g \leq f$, then

$$
\iota(f, g)=\bigcap_{r \in \mathbb{Q}}(f(-, r) \vee g(r,-)) \supseteq \bigcap_{r \in \mathbb{Q}}(f(-, r) \vee f(r,-))=L .
$$

Conversely, suppose that $\iota(f, g)=L$, that is, $f(-, q) \vee g(q,-)=L$ for all $q \in \mathbb{Q}$. If $q>r$, then $f(r,-) \cap f(-, q)=\{1\}$ and thus

$$
\begin{aligned}
f(r,-) & \subseteq f(r,-) \vee g(q,-) \\
& =(f(r,-) \vee g(q,-)) \cap(f(-, q) \vee g(q,-)) \\
& =(f(r,-) \cap f(-, q)) \vee g(q,-) \\
& =g(q,-) .
\end{aligned}
$$

Hence $f(r,-) \subseteq \bigcap_{q>r} g(q,-)=g(r,-)$, that is, $g \leq f$.

(2) If $f<g$, then $f \leq g$ (as shown in [9]) and $\iota(f, g)=\{1\}$ (by definition). Hence, $\iota(f, g) \neq L$, so that $g \not \leq f$ (by $(1))$. In particular, $f \supsetneqq g$.

(3) If $f<g$ and $g<h$ then $(g-f)(0,-)=\iota(\mathbf{0}, g-f)=\iota(f, g)=\{1\}$ and, similarly, $(h-g)(0,-)=\{1\}$. Hence

$$
\iota(f, h)=(h-f)(0,-)=(h-g)(0,-) \vee(g-h)(0,-)=\{1\} .
$$

Now, let $X$ be a topological space. If $G: X \rightarrow \mathbb{R}$ is a lower semicontinuous function that dominates an upper semicontinuous function $F: X \rightarrow \mathbb{R}$ (in the sense that $F \leq G)$, the family $\left\{\mathfrak{c}\left(G^{-1}(r, \infty)\right)\right\}_{r \in \mathbb{Q}}$ is clearly a scale (the assumption that $G$ dominates an upper semicontinuous function is essential here; see [14]). It consequently determines a lower semicontinuous $\varphi_{G}: \mathfrak{L}(\mathbb{R}) \rightarrow \mathcal{S}(\Omega(X))^{\text {op }}$, defined by

$$
\varphi_{G}(r,-)=\mathfrak{c}\left(G^{-1}(r,+\infty)\right) \quad \text { and } \quad \varphi_{G}(-, r)=\bigcap_{q<r} \mathfrak{o}\left(G^{-1}(q,+\infty)\right) .
$$

Dually, for any upper semicontinuous $F: X \rightarrow \mathbb{R}$ dominated by a lower semicontinuous $G: X \rightarrow \mathbb{R}$, the system $\left\{\mathfrak{o}\left(F^{-1}(-\infty, r)\right)\right\}_{r \in \mathbb{Q}}$ is a scale that determines an upper semicontinuous $\varphi_{F}: \mathfrak{L}(\mathbb{R}) \rightarrow \mathcal{S}(\Omega(X))^{\mathrm{o} p}$ with

$$
\varphi_{F}(r,-)=\bigcap_{q>r} \mathfrak{o}\left(F^{-1}(-\infty, q)\right) \quad \text { and } \quad \varphi_{F}(-, r)=\mathfrak{c}\left(F^{-1}(-\infty, r)\right) .
$$

In this situation it is easy to check that $F \leq G$ if and only if $\varphi_{F} \leq \varphi_{G}$. Moreover, we have the following:

Proposition 3.4. Let $X$ be a topological space. For any $F, G: X \rightarrow \mathbb{R}, F$ upper semicontinuous and $G$ lower semicontinuous, such that $F \leq G$, we have:

(1) $\iota\left(\varphi_{F}, \varphi_{G}\right)=\mathfrak{c}\left((G-F)^{-1}(0,+\infty)\right)$.

(2) $F<G$ iff $\varphi_{F}<\varphi_{G}$. 
Proof. (1) Since $x \in \bigcup_{r \in \mathbb{Q}} F^{-1}(-\infty, r) \cap G^{-1}(r, \infty)$ iff $F(x)<G(x)$, we have

$$
\begin{aligned}
\iota\left(\varphi_{F}, \varphi_{G}\right) & =\bigcap_{r \in \mathbb{Q}}\left(\varphi_{F}(-, r) \vee \varphi_{G}(r,-)\right) \\
& =\bigcap_{r \in \mathbb{Q}}\left(\mathfrak{c}\left(F^{-1}(-\infty, r)\right) \vee \mathfrak{c}\left(G^{-1}(r, \infty)\right)\right) \\
& =\mathfrak{c}\left(\bigcup_{r \in \mathbb{Q}} F^{-1}(-\infty, r) \cap G^{-1}(r, \infty)\right) \\
& =\mathfrak{c}\left((G-F)^{-1}(0,+\infty)\right) .
\end{aligned}
$$

(2) follows immediately from (1) (see also [9, Remark 6.5]).

Recall that a topological space $X$ is $T_{D}([1])$, a separation condition stronger than $T_{0}$ but weaker than $T_{1}$, if points are locally closed, that is, for every $x \in X$ there is an open set $U \ni x$ such that $U \backslash\{x\}$ is still open.

Proposition 3.5. Let $X$ be a topological space. For any upper semicontinuous $F: X \rightarrow \mathbb{R}$ such that $0 \leq F$ we have:

(1) If $F^{-1}(0,+\infty) \in \Omega(X)$, then $\iota\left(\mathbf{0}, \varphi_{F}\right)^{\#}=\mathfrak{o}\left(F^{-1}(0,+\infty)\right)$.

(2) If $0<F$, then $\mathbf{0}<\varphi_{F}$.

Moreover, if $X$ is $T_{D}$ then:

(3) If $\iota\left(\mathbf{0}, \varphi_{F}\right)^{\#}=\mathfrak{o}(U)$ for some $U \in \Omega(X)$, then $U=F^{-1}(0,+\infty)$.

(4) $0<F$ iff $\quad \mathbf{0}<\varphi_{F}$.

Proof. (1) Since $F^{-1}(-\infty, r) \cup F^{-1}(0, \infty)=X$ for all $r>0$, it follows that

$$
\iota\left(\mathbf{0}, \varphi_{F}\right)^{\#}=\varphi_{F}(0,-)^{\#}=\bigvee_{r>0} \mathfrak{c}\left(F^{-1}(-\infty, r)\right) \subseteq \mathfrak{o}\left(F^{-1}(0, \infty)\right) .
$$

On the other hand, let $V \in \mathfrak{o}\left(F^{-1}(0, \infty)\right)$ and $V_{r}=F^{-1}(-\infty, r) \cup V$ for each $r>0$. Then $V_{r} \in \mathfrak{c}\left(F^{-1}(-\infty, r)\right)$ and

$$
\begin{aligned}
V & =F^{-1}(0, \infty) \rightarrow V=\operatorname{Int}\left(F^{-1}(-\infty, 0] \cup V\right) \\
& =\operatorname{Int}\left(\left(\bigcap_{r>0} F^{-1}(-\infty, r)\right) \cup V\right)=\operatorname{Int}\left(\bigcap_{r>0} V_{r}\right)=\bigwedge_{r>0} V_{r} .
\end{aligned}
$$

Hence $V \in \bigvee_{r>0} \mathfrak{c}\left(F^{-1}(-\infty, r)\right)=\iota\left(\mathbf{0}, \varphi_{F}\right)^{\#}$.

(2) follows immediately from (1).

(3) Since $\bigvee_{r>0} \mathfrak{c}\left(F^{-1}(-\infty, r)\right)=\iota\left(\mathbf{0}, \varphi_{F}\right)^{\#}=\mathfrak{o}(U)$, we have $F^{-1}(-\infty, r) \cup$ $U=X$ for every $r>0$. Hence $F^{-1}(0,+\infty)=\bigcup_{r>0} F^{-1}[r,+\infty) \subseteq U$.

On the other hand, consider $x \in U$. Since $X$ is $T_{D}$, there is an open $V \ni x$ such that $W=V \backslash\{x\}$ is open as well. Moreover, since $U \rightarrow W \in$ $\mathfrak{o}(U)=\bigvee_{r>0} \mathfrak{c}\left(F^{-1}(-\infty, r)\right)$, there exists a countable family $\left(V_{r}\right)_{r>0}$ in $\Omega(X)$ such that $F^{-1}(-\infty, r) \subseteq V_{r}$ for each $r>0$ and

$$
U \rightarrow W=\bigwedge_{r>0} V_{r}=\operatorname{Int}\left(\bigcap_{r>0} V_{r}\right)
$$


Then $U \cap V \nsubseteq W$ (because $x \in U \cap V)$ and thus $V \nsubseteq U \rightarrow W$, from which it follows that $x \notin U \rightarrow W$ (because $V \backslash\{x\}=W \subseteq U \rightarrow W$ ). Hence

$$
x \in X \backslash \operatorname{Int}\left(\bigcap_{r>0} V_{r}\right)=\overline{\bigcup_{r>0}\left(X \backslash V_{r}\right)} .
$$

Since $V$ is an open neighborhood of $x$ it follows that $V \cap\left(\bigcup_{r>0}\left(X \backslash V_{r}\right)\right) \neq \varnothing$. But $W \subseteq U \rightarrow W \subseteq \bigcap_{r>0} V_{r}$. Hence

$$
x \in \bigcup_{r>0}\left(X \backslash V_{r}\right) \subseteq \bigcup_{r>0}\left(X \backslash F^{-1}(-\infty, r)\right)=F^{-1}(0,+\infty) .
$$

(4) follows immediately from (2) and (3).

Note that, in general, it is false that $0<F$ iff $\mathbf{0}<\varphi_{F}$. A counterexample can be obtained from Proposition 3.3 of [7]:

Let $\mathbb{N}$ be endowed with the cofinite topology and let $F: \mathbb{N} \rightarrow \mathbb{R}$ be given by $F(n)=\frac{1}{n}$ for each $n \in \mathbb{N}$. Clearly, $F$ is upper semicontinuous and $0<F$. Nevertheless,

$$
\begin{aligned}
\iota\left(\mathbf{0}, \varphi_{F}\right) & =\bigcap_{r>0} \mathfrak{o}\left(F^{-1}(-\infty, r)\right)=\bigcap_{n \in \mathbb{N}} \mathfrak{o}(\mathbb{N} \backslash\{1, \ldots, n\})= \\
& =\bigcap_{n \in \mathbb{N}}(\{\varnothing\} \cup\{V \in \Omega(\mathbb{N}) \mid\{1, \ldots, n\} \subseteq V\})=\{\varnothing, \mathbb{N}\} \neq\{\mathbb{N}\} .
\end{aligned}
$$

Corollary 3.6. Let $X$ be a topological space. For any $F, G: X \rightarrow \mathbb{R}, F$ upper semicontinuous and $G$ lower semicontinuous, such that $G \leq F$, we have:

(1) If $(F-G)^{-1}(0,+\infty) \in \Omega(X)$, then $\iota\left(\varphi_{G}, \varphi_{F}\right)^{\#}=\mathfrak{o}\left((F-G)^{-1}(0,+\infty)\right)$.

(2) If $G<F$, then $\varphi_{G}<\varphi_{F}$.

Moreover, if $X$ is $T_{D}$ then:

(3) If $\iota\left(\varphi_{G}, \varphi_{F}\right)^{\#}=\mathfrak{o}(U)$ for some $U \in \Omega(X)$, then $U=(F-G)^{-1}(0,+\infty)$.

(4) $G<F$ iff $\varphi_{G}<\varphi_{F}$.

Proof. First, check that $\varphi_{(F-G)}=\varphi_{F}-\varphi_{G}$. Then use Proposition 3.5.

Corollary 3.7. Let $X$ be a topological space and let $F, G: X \rightarrow \mathbb{R}, F$ upper semicontinuous and $G$ lower semicontinuous, such that $0 \leq F \leq G$. Furthermore, let $U=G^{-1}(0,+\infty)$. Then:

(1) If $0<\left.F\right|_{U}<\left.G\right|_{U}$, then $\iota\left(\mathbf{0}, \varphi_{G}\right)^{\#}=\iota\left(\mathbf{0}, \varphi_{F}\right)^{\#}=\iota\left(\varphi_{F}, \varphi_{G}\right)^{\#}$.

Moreover, if $X$ is a $T_{D}$-space then:

(2) $0<\left.F\right|_{U}<\left.G\right|_{U} \quad$ iff $\quad \iota\left(\mathbf{0}, \varphi_{G}\right)^{\#}=\iota\left(\mathbf{0}, \varphi_{F}\right)^{\#}=\iota\left(\varphi_{F}, \varphi_{G}\right)^{\#}$.

Proof. (1) If $0<\left.F\right|_{U}<\left.G\right|_{U}$ then

$$
U \subseteq\{x \in X \mid 0<F(x)<G(x)\}=F^{-1}(0,+\infty) \cap(G-F)^{-1}(0,+\infty)
$$

and hence it follows from Proposition 3.5 (1), Proposition 3.4 (1), and Corollary $3.6(1)$ that

$$
\begin{aligned}
\iota\left(\mathbf{0}, \varphi_{G}\right)^{\#}=\mathfrak{o}(U) & \subseteq \mathfrak{o}\left(F^{-1}(0,+\infty)\right) \cap \mathfrak{o}\left((G-F)^{-1}(0,+\infty)\right) \\
& =\iota\left(\mathbf{0}, \varphi_{F}\right)^{\#} \cap \iota\left(\varphi_{F}, \varphi_{G}\right)^{\#} .
\end{aligned}
$$

On the other hand, since $\mathbf{0} \leq \varphi_{F} \leq \varphi_{G}$, property 3.1 (4) implies that $\iota\left(\mathbf{0}, \varphi_{F}\right)^{\#} \subseteq \iota\left(\mathbf{0}, \varphi_{G}\right)^{\#}$ and $\iota\left(\varphi_{F}, \varphi_{G}\right)^{\#} \subseteq \iota\left(\mathbf{0}, \varphi_{G}\right)^{\#}$. 
(2) Let $X$ be a $T_{D}$-space and assume that

$$
\iota\left(\mathbf{0}, \varphi_{G}\right)^{\#}=\iota\left(\mathbf{0}, \varphi_{F}\right)^{\#}=\iota\left(\varphi_{F}, \varphi_{G}\right)^{\#} .
$$

By Proposition $3.4(1), \iota\left(\mathbf{0}, \varphi_{G}\right)^{\#}=\mathfrak{o}(U)$ and hence it follows from Proposition $3.5(3)$ and Corollary $3.6(3)$ that $U=F^{-1}(0,+\infty)=(G-F)^{-1}(0,+\infty)$. Consequently, $\mathbf{0}<\left.F\right|_{U}<\left.G\right|_{U}$.

\section{Strict insertion results for perfect locales}

Various predecessors of the following insertion lemma can be found in $[3,4,5]$.

Lemma 4.1. Let $L$ be a locale and $f \in \mathrm{F}(L)$ with $\mathbf{0} \leq f$. Further, let $\left\{a_{n}\right\}_{n \in \mathbb{N}}$ be an antitone sequence in $L$ such that

(1) $\left.\mathfrak{c}\left(a_{n}\right) \subseteq f\left(\frac{1}{n},-\right)\right)^{\#}$ for all $n$, and

(2) $\bigvee_{n \in \mathbb{N}} \mathfrak{c}\left(a_{n}\right) \supseteq f(0,-)^{\#}$.

Then there exists an upper semicontinuous $u \in \mathrm{F}(L)$ such that

$$
\mathbf{0} \leq u \leq f \quad \text { and } \quad \iota(\mathbf{0}, f)^{\#}=\iota(\mathbf{0}, u)^{\#}=\iota(u, f)^{\#} .
$$

Moreover, if $f$ satisfies the stronger condition

$\left(2^{\prime}\right) \bigcap_{n \in \mathbb{N}} \mathfrak{o}\left(a_{n}\right) \subseteq f(0,-)$,

then $\iota(\mathbf{0}, f)=\iota(\mathbf{0}, u)=\iota(u, f)$.

Note. It is important to note here that if $\iota(\mathbf{0}, f)=\iota(\mathbf{0}, u)=\iota(u, f)$ then $\iota(\mathbf{0}, f)^{\#}=\iota(\mathbf{0}, u)^{\#}=\iota(u, f)^{\#}$, but not conversely.

Proof. For each $r \in \mathbb{Q}$ define a sublocale $S_{r}$ of $L$ as follows:

$$
S_{r}= \begin{cases}\{1\} & \text { if } r \leq 0 \\ \mathfrak{o}\left(a_{n}\right) & \text { if } \frac{1}{2(n+1)} \leq r<\frac{1}{2 n} \\ L & \text { if } r \geq \frac{1}{2} .\end{cases}
$$

Since each $S_{r}$ is complemented, in order to conclude that $\left\{S_{r}\right\}_{r \in \mathbb{Q}}$ is a scale it suffices to check that it is isotone (recall Remark 2.1). Let $r<q$ in $\mathbb{Q}$. If $r \leq 0$ or $q \geq \frac{1}{2}$ then, obviously, $S_{r} \subseteq S_{q}$. Otherwise, for $0<r<q<\frac{1}{2}$ we consider the following two cases:

- if $\frac{1}{2(n+1)} \leq r<q<\frac{1}{2 n}$ for some $n$, then

$$
\begin{gathered}
S_{r}=\mathfrak{o}\left(a_{n}\right)=S_{q} \\
- \text { if } \frac{1}{2(n+1)} \leq r<\frac{1}{2 n} \leq \frac{1}{2(m+1)} \leq q<\frac{1}{2 m} \text { for some } m<n, \text { then } \\
S_{r}=\mathfrak{o}\left(a_{n}\right) \subseteq \mathfrak{o}\left(a_{m}\right)=S_{q} .
\end{gathered}
$$

Thus, $\left\{S_{r}\right\}_{r \in \mathbb{Q}}$ determines a $u \in \mathrm{F}(L)$ defined by

$$
u(r,-)=\bigcap_{q>r} S_{q} \text { and } u(-, r)=\bigcap_{q<r} S_{q}^{\#}
$$


for every $r \in \mathbb{Q}$. More specifically,

$$
u(r,-)= \begin{cases}\{1\} & \text { if } r<0 \\ \bigcap_{n \in \mathbb{N}} \mathfrak{o}\left(a_{n}\right) & \text { if } r=0 \\ \mathfrak{o}\left(a_{n}\right) & \text { if } \frac{1}{2(n+1)} \leq r<\frac{1}{2 n} \\ L & \text { if } r \geq \frac{1}{2}\end{cases}
$$

and

$$
u(-, r)= \begin{cases}L & \text { if } r \leq 0 \\ \mathfrak{c}\left(a_{n}\right) & \text { if } \frac{1}{2(n+1)}<r \leq \frac{1}{2 n} \\ \{1\} & \text { if } r>\frac{1}{2},\end{cases}
$$

and $u$ is clearly upper semicontinuous. Moreover, $\mathbf{0} \leq u \leq 2 u$, since $u(r,-)=$ $\{1\}=\mathbf{0}(r,-)$ for all $r<0$ and $(2 u)(r,-)=u\left(\frac{r}{2},-\right) \subseteq u(r,-)$ for all $r$. To see that $2 u \leq f$, we first note that $f(r,-) \subseteq f(s,-)^{\# \#}$ for each $r<s$ in $\mathbb{Q}$ and distinguish the following four cases:

- if $r<0$, then $f(r,-)=\{1\} \subseteq(2 u)(r,-)$;

- if $r \geq 1$, then $f(r,-) \subseteq L=u\left(\frac{r}{2},-\right)=(2 u)(r,-)$;

- if $r=0$, then by assumption (1) we obtain

$$
f(r,-)=f(0,-) \subseteq \bigcap_{n \in \mathbb{N}} f\left(\frac{1}{n},-\right)^{\# \#} \subseteq \bigcap_{n \in \mathbb{N}} \mathfrak{o}\left(a_{n}\right)=u(0,-)=(2 u)(r,-) ;
$$

- if $\frac{1}{n+1} \leq r<\frac{1}{n}$, then by assumption (1) we have

$$
f(r,-) \subseteq f\left(\frac{1}{n},-\right)^{\# \#} \subseteq \mathfrak{o}\left(a_{n}\right)=u\left(\frac{r}{2},-\right)=(2 u)(r,-) .
$$

In any case, $f(r,-) \subseteq(2 u)(r,-)$. Hence $2 u \leq f$, i.e. $u \leq f-u$ and thus $\iota(\mathbf{0}, u) \supseteq \iota(\mathbf{0}, f-u)=\iota(u, f)$. Moreover, by assumption $(2)$,

$\iota(\mathbf{0}, f)^{\#}=f(0,-)^{\#} \subseteq \bigvee_{n \in \mathbb{N}} \mathfrak{c}\left(a_{n}\right)=u(0,-)^{\#}=\iota(\mathbf{0}, u)^{\#} \subseteq \iota(u, f)^{\#} \subseteq \iota(\mathbf{0}, f)^{\#}$.

Finally, if $f$ satisfies the stronger condition $\left(2^{\prime}\right)$, we get

$$
\iota(\mathbf{0}, f)=f(0,-) \supseteq \bigcap_{n \in \mathbb{N}} \mathfrak{o}\left(a_{n}\right)=u(0,-)=\iota(\mathbf{0}, u) \supseteq \iota(u, f) \supseteq \iota(\mathbf{0}, f) .
$$

Recall that a locale $L$ is perfect if

every open sublocale is a countable join of closed ones

(formulated in the coframe $\mathcal{S}(L)$ of sublocales).

For any perfect space $X$, the locale $\Omega(X)$ is perfect [7, Proposition 3.6]. For more information on localic perfectness we refer to [5, Section 4$]$ and $[7$, Remarks 3.10].

In the proof of the next result we shall use the characteristic function of an open sublocale $\mathfrak{o}(a)$, that is, the lower semicontinuous function $\chi_{\mathfrak{o}(a)} \in$ $\mathrm{F}(L)$ defined by

$$
\chi_{\mathfrak{o}(a)}(r,-)= \begin{cases}\{1\} & \text { if } r<0 \\ \mathfrak{c}(a) & \text { if } 0 \leq r<1 \\ L & \text { if } r \geq 1\end{cases}
$$


and

$$
\chi_{\mathfrak{o}(a)}(-, r)= \begin{cases}L & \text { if } r \leq 0 \\ \mathfrak{o}(a) & \text { if } 0<r \leq 1 \\ \{1\} & \text { if } r>1 .\end{cases}
$$

Proposition 4.2. The following statements are equivalent for a locale L:

(1) $L$ is perfect.

(2) For each lower semicontinuous $l \in \mathrm{F}(L)$ such that $\mathbf{0} \leq l$, there exists an upper semicontinuous $u \in \mathrm{F}(L)$ such that

$$
\mathbf{0} \leq u \leq l \quad \text { and } \quad \iota(\mathbf{0}, l)^{\#}=\iota(\mathbf{0}, u)^{\#}=\iota(u, l)^{\#} .
$$

Proof. $(1) \Rightarrow(2)$ : Let $l \in \mathrm{F}(L)$ be lower semicontinuous with $\mathbf{0} \leq l$. For each natural $n$ there exists a $b_{n} \in L$ such that

$$
l\left(\frac{1}{n},-\right)=\mathfrak{c}\left(b_{n}\right) .
$$

Then, by perfectness of $L$, there exists a family $\left\{b_{n m}\right\}_{m \in \mathbb{N}}$, for each $n$, such that

$$
\mathfrak{o}\left(b_{n}\right)=\bigvee_{m \in \mathbb{N}} \mathfrak{c}\left(b_{n m}\right)
$$

Put

$$
a_{n}=\bigwedge_{i, j \leq n} b_{i j}
$$

Then $\left\{a_{n}\right\}_{n \in \mathbb{N}}$ is antitone and

$$
\mathfrak{c}\left(a_{n}\right)=\bigvee_{i, j \leq n} \mathfrak{c}\left(b_{i j}\right) \subseteq \bigvee_{i \leq n} \bigvee_{m \in \mathbb{N}} \mathfrak{c}\left(b_{i m}\right)=\bigvee_{i \leq n} \mathfrak{o}\left(b_{i}\right)=\bigvee_{i \leq n} l\left(\frac{1}{i},-\right)^{\#}=l\left(\frac{1}{n},-\right)^{\#}
$$

Moreover, $\mathfrak{o}\left(b_{n}\right)=l\left(\frac{1}{n},-\right)^{\#} \supseteq l(0,-)^{\#}$ for all $n$ and thus

$$
\bigvee_{n \in \mathbb{N}} \mathfrak{c}\left(a_{n}\right)=\bigvee_{n \in \mathbb{N}} \bigvee_{i, j \leq n} \mathfrak{c}\left(b_{i j}\right)=\bigvee_{n, m \in \mathbb{N}} \mathfrak{c}\left(b_{n m}\right)=\bigvee_{n \in \mathbb{N}} \mathfrak{o}\left(b_{n}\right) \supseteq l(0,-)^{\#}
$$

The required assertion follows then from Lemma 4.1.

$(2) \Rightarrow(1)$ : Let $a \in L$. Since $\chi_{\mathfrak{o}(a)}$ is lower semicontinuous, there exists an upper semicontinuous $u$ with $\mathbf{0} \leq u \leq \chi_{\mathfrak{o}(a)}$ and $\iota\left(\mathbf{0}, \chi_{\mathfrak{o}(a)}\right)^{\#}=\iota(\mathbf{0}, u)^{\#}=$ $\iota\left(u, \chi_{\mathfrak{o}(a)}\right)^{\#}$.

$$
\mathfrak{o}(a)=\chi_{\mathfrak{o}(a)}(0,-)^{\#}=\iota\left(\mathbf{0}, \chi_{\mathfrak{o}(a)}\right)^{\#}=\iota(\mathbf{0}, u)^{\#}=u(0,-)^{\#}=\bigvee_{n \in \mathbb{N}} u\left(-, \frac{1}{n}\right)
$$

is a countable join of closed sublocales. Hence $L$ is perfect.

Corollary 4.3. The following statements are equivalent for a $T_{D}$-space $X$ :

(1) $X$ is perfect.

(2) If $F, G: X \rightarrow \mathbb{R}$ are such that $F \leq G$, with $F$ upper semicontinuous and $G$ lower semicontinuous, then there exist $F^{\prime}, G^{\prime}: X \rightarrow \mathbb{R}, F^{\prime}$ upper semicontinuous and $G^{\prime}$ lower semicontinuous, such that $F \leq F^{\prime} \leq G^{\prime} \leq$ $G$ and $F(x)<F^{\prime}(x)<G^{\prime}(x)<G(x)$ whenever $F(x)<G(x)$. 
Proof. We only prove $(1) \Rightarrow(2)$. Let $X$ be a perfect space and let $F, G: X \rightarrow \mathbb{R}$ be such that $F \leq G, F$ is upper semicontinuous and $G$ is lower semicontinuous. Then $\Omega(X)$ is a perfect locale and

$$
l=\varphi_{(G-F)} \in \mathrm{F}(\Omega(X))
$$

is lower semicontinuous with $\mathbf{0} \leq l$. Then, by Proposition 4.2 , there exists an upper semicontinuous function $u$ such that

$$
\mathbf{0} \leq u \leq l \quad \text { and } \quad \iota(\mathbf{0}, l)^{\#}=\iota(\mathbf{0}, u)^{\#}=\iota(u, l)^{\#},
$$

and by Proposition $3.4(1), \iota(\mathbf{0}, l)^{\#}=\mathfrak{o}\left((G-F)^{-1}(0,+\infty)\right)$.

Let $u(-, r)=\mathfrak{c}\left(U_{r}\right)$ and define $H: X \rightarrow \mathbb{R}$ by

$$
H(x)=\bigwedge\left\{r \in \mathbb{Q} \mid x \in U_{r}\right\} .
$$

Then $H$ is upper semicontinuous, $0 \leq H \leq G-F$ and $\varphi_{H}=u$. Since $X$ is a $T_{D}$-space, it follows from Corollary $3.7(2)$ that

$$
0<\left.H\right|_{(G-F)^{-1}(0,+\infty)}<\left.(G-F)\right|_{(G-F)^{-1}(0,+\infty)},
$$

that is, $0<H(x)<G(x)-F(x)$ whenever $F(x)<G(x)$.

Finally, let $F^{\prime}=F+\frac{H}{2}$ and $G^{\prime}=G-\frac{H}{2}$. Then $F^{\prime}$ is upper semicontinuous, $G^{\prime}$ is lower semicontinuous and $F \leq F^{\prime} \leq G^{\prime} \leq G$. Moreover, if $F(x)<G(x)$ then

$$
F(x)<F(x)+\frac{H(x)}{2}=F^{\prime}(x) \leq G^{\prime}(x)=G(x)-\frac{H(x)}{2}<G(x) .
$$

We may now present our main result.

Theorem 4.4. The following statements are equivalent for a locale L:

(1) $L$ is perfect.

(2) For any upper semicontinuous $u \in \mathrm{F}(L)$ and any lower semicontinuous $l \in \mathrm{F}(L)$ such that $u \leq l$, there exist $u^{\prime}, l^{\prime} \in \mathrm{F}(L)$, $u^{\prime}$ upper and $l^{\prime}$ lower semicontinuous, such that

$$
u \leq u^{\prime} \leq l^{\prime} \leq l \quad \text { and } \quad \iota(u, l)^{\#}=\iota\left(u, u^{\prime}\right)^{\#}=\iota\left(u^{\prime}, l^{\prime}\right)^{\#}=\iota\left(l^{\prime}, l\right)^{\#} .
$$

Proof. $(1) \Rightarrow(2)$ : Let us assume that $L$ is perfect, and that $u, l \in \mathrm{F}(L)$ satisfy the assumption in (2). Then $l-u$ is lower semicontinuous and $\mathbf{0} \leq l-u$. By Proposition 4.2, there exists an upper semicontinuous $u_{1}$ such that

$$
\mathbf{0} \leq u_{1} \leq l-u \quad \text { and } \quad \iota(\mathbf{0}, l-u)^{\#}=\iota\left(\mathbf{0}, u_{1}\right)^{\#}=\iota\left(u_{1}, l-u\right)^{\#} .
$$

Then $u^{\prime}=u+u_{1}$ is again upper semicontinuous and we have

$$
u \leq u^{\prime} \leq l \quad \text { and } \quad \iota(u, l)^{\#}=\iota\left(u, u^{\prime}\right)^{\#}=\iota\left(u^{\prime}, l\right)^{\#} .
$$

By a similar argument, since $u^{\prime}$ is upper semicontinuous, $l$ is lower semicontinuous and $\mathbf{0} \leq l-u^{\prime}$, then there exists an upper semicontinuous $u_{2}$ such that

$$
\mathbf{0} \leq u_{2} \leq l-u^{\prime} \quad \text { and } \quad \iota\left(\mathbf{0}, l-u^{\prime}\right)^{\#}=\iota\left(\mathbf{0}, u_{2}\right)^{\#}=\iota\left(u_{2}, l-u^{\prime}\right)^{\#} .
$$

Then $l^{\prime}=l-u_{2}$ is lower semicontinuous and we have

$$
u \leq u^{\prime} \leq l^{\prime} \leq l \quad \text { and } \quad \iota(u, l)^{\#}=\iota\left(u, u^{\prime}\right)^{\#}=\iota\left(u^{\prime}, l\right)^{\#}=\iota\left(l^{\prime}, l\right)^{\#} .
$$

$(2) \Rightarrow(1)$ follows from Proposition 4.2. 
In the next section we will analyse the effect of the normal property of a locale on the insertion property of Theorem 4.4.

\section{Michael's insertion theorem revisited}

Recall the property of perfectness of locales. The dual property that

every closed sublocale is a countable join of open ones

looks deceptively equivalent to it. Actually, however, it is stronger (since $\mathcal{S}(L)$ is not Boolean in general). In [7], we called it the $G_{\delta^{-}}$perfect property and observed that $G_{\delta}$-perfect and perfect properties do coincide under normality. It is also worth pointing that $G_{\delta}$-perfect sublocales of compact regular locales are spatial (see [15, VII.7.3]).

An easy reformulation of the proofs of 4.2 and 4.4 yields the following characterization of $G_{\delta}$-perfectness.

Theorem 5.1. The following statements are equivalent for a locale L:

(1) $L$ is $G_{\delta}$-perfect.

(2) For each lower semicontinuous $l \in \mathrm{F}(L)$ such that $\mathbf{0} \leq l$, there exists an upper semicontinuous $u \in \mathrm{F}(L)$ such that

$$
\mathbf{0} \leq u \leq l \quad \text { and } \quad \iota(\mathbf{0}, l)=\iota(\mathbf{0}, u)=\iota(u, l) .
$$

(3) For any upper semicontinuous $u \in \mathrm{F}(L)$ and any lower semicontinuous $l \in \mathrm{F}(L)$ such that $u \leq l$, there exist $u^{\prime}, l^{\prime} \in \mathrm{F}(L)$, $u^{\prime}$ upper and $l^{\prime}$ lower semicontinuous, such that

$$
u \leq u^{\prime} \leq l^{\prime} \leq l \quad \text { and } \quad \iota(u, l)=\iota\left(u, u^{\prime}\right)=\iota\left(u^{\prime}, l^{\prime}\right)=\iota\left(l^{\prime}, l\right) .
$$

Recall now that a locale $L$ is normal if for any $a, b \in L$ with $a \vee b=1$, there exist $u, v \in L$ such that $a \vee u=1=b \vee v$ and $u \wedge v=0$. The following is the pointfree counterpart of the celebrated Katětov-Tong insertion theorem, formulated as in [6].

Theorem 5.2. A locale $L$ is normal if and only if for any upper semicontinuous $u \in \mathrm{F}(L)$ and any lower semicontinuous $l \in \mathrm{F}(L)$ such that $u \leq l$, there exists a continuous $f \in \mathrm{F}(L)$ such that $u \leq f \leq l$.

Combining normality and perfectness, we have the notion of a perfectly normal locale ([5]). As mentioned before, this is the same as normal plus $G_{\delta}$-perfect (see [7] for the details). Then Theorem 5.2 plus Theorem 5.1 yield the pointfree version of Michael's insertion theorem formulated exactly as in [9] (see also [5]).

Theorem 5.3. A locale $L$ is perfectly normal if and only if for any upper semicontinuous $u \in \mathrm{F}(L)$ and any lower semicontinuous $l \in \mathrm{F}(L)$ such that $u \leq l$, there exists a continuous $f \in \mathrm{F}(L)$ such that

$$
u \leq f \leq l \quad \text { and } \quad \iota(u, f)=\iota(f, l)=\iota(u, l) .
$$


Proof. $\Rightarrow$ : Let $u \leq l$ in $\mathrm{F}(L)$ with $u$ upper semicontinuous and $l$ lower semicontinuous. By the double insertion property in 5.1, there exist $u^{\prime}, l^{\prime} \in \mathrm{F}(L)$, $u^{\prime}$ upper and $l^{\prime}$ lower semicontinuous, such that

$$
u \leq u^{\prime} \leq l^{\prime} \leq l \quad \text { and } \quad \iota(u, l)=\iota\left(u, u^{\prime}\right)=\iota\left(u^{\prime}, l^{\prime}\right)=\iota\left(l^{\prime}, l\right) .
$$

Then, by 5.2 , there is a continuous $f \in \mathrm{F}(L)$ such that $u^{\prime} \leq f \leq l^{\prime}$.

Finally, by property $3.1(4), \iota(u, l) \subseteq \iota(u, f)$ and $\iota(u, f) \subseteq \iota\left(u, u^{\prime}\right)=\iota(u, l)$, that is, $\iota(u, f)=\iota(u, l)$. Similarly, $\iota(f, l)=\iota(u, l)$.

$\Leftarrow$ : The normality of $L$ follows immediately from the assumption (by 5.2 ). In order to show that $L$ is $G_{\delta}$-perfect let $u, l \in \mathrm{F}(L), u \leq l$, with $u$ upper semicontinuous and $l$ lower semicontinuous, and take the continuous $f$ provided by the assumption. Then define $u^{\prime}$ and $l^{\prime}$ by

$$
u^{\prime}(-, r)=u(-, r), u^{\prime}(r,-)=f(r,-)
$$

and

$$
l^{\prime}(-, r)=f(-, r), l^{\prime}(r,-)=l(r,-) .
$$

It is a routine exercise to check that $u^{\prime}$ and $l^{\prime}$ are indeed in $\mathrm{F}(L)$ and satisfy all conditions in $5.1(3)$.

\section{Appendix: strict insertion for $\mathcal{A}$-semicontinuities}

The paper [10] proposes a unified treatment of several variants of semicontinuities and continuities and their insertion results, viz. $\mathcal{A}$-continuity of an $f \in \mathrm{F}(L)$ where $\mathcal{A}$ is a class of complemented sublocales of $L$. We end this paper with some notes about the application of this approach to the results above. To simplify we will only consider classes $\mathcal{A}=\{\mathfrak{c}(a) \mid a \in A\}$ for some $A \subseteq L$. In what follows we shall speak about $A$ being normal or perfect in $L$.

In this terminology (see [8] for more information and examples), an $A \subseteq L$ is called normal in $L$ if for any $a, b \in A$ with $a \vee b=1$ there exist $u, v \in A$ such that $a \vee u=1=b \vee v$ and $u \wedge v=0$. An $f \in \mathrm{F}(L)$ is lower A-semicontinuous if, whenever $r<q$ in $\mathbb{Q}$, there is an $a \in A$ such that $f(r,-) \subseteq \mathfrak{c}(a) \subseteq f(q,-)$. Analogously, $f$ is upper A-semicontinuous if, whenever $r<q$ in $\mathbb{Q}$, there is an $a \in A$ such that $f(-, q) \subseteq \mathfrak{c}(a) \subseteq f(-, r)$, and $f$ is $A$-continuous if it is both lower and upper $A$-semicontinuous.

The case $A=L$ yields the usual notions of normality and lower and upper semicontinuities. For $A=\left\{a \in L \mid a^{* *}=a\right\}$ (that is, the set of all regular elements of $L), A$ is normal in $L$ if and only if $L$ is mildly normal. In this case, lower (resp., upper) $A$-semicontinuity means normal lower (resp., normal upper) semicontinuity. On the other hand, the set $A=\operatorname{Coz} L$ of all cozero elements of $L$ is normal in $L$. In this case, lower (resp., upper) $A$-semicontinuity means zero lower (resp., upper) semicontinuity.

Following this idea, we say that $A \subseteq L$ is perfect (resp. $G_{\delta}$-perfect) in $L$ if

for each $a \in A$ there is a countable $B \subseteq A$ such that $\mathfrak{o}(a)=$ $\bigvee_{b \in B} \mathfrak{c}(b)\left(\operatorname{resp} . \mathfrak{c}(a)=\bigcap_{b \in B} \mathfrak{o}(b)\right)$. 
A careful scrutiny of the proofs of 4.2 and 4.4 shows that they continue to hold for $A$-semicontinuous functions in case $A$ is closed under finite meets and $0 \in A$. Similarly, the version of 5.1 for $A$-semicontinuous functions requires that $A$ is closed under finite joins and $1 \in A$. We know further from [10] that 5.2 holds for $A$-normal locales whenever $A$ is a sublattice of $L$. Hence we have:

Theorem 1. Let $L$ be a locale and let $A \subseteq L$ be closed under finite meets with $0 \in A$. Then the following statements are equivalent:

(1) $A$ is perfect in $L$.

(2) For any upper A-semicontinuous $u \in \mathrm{F}(L)$ and any lower A-semicontinuous $l \in \mathrm{F}(L)$ such that $u \leq l$, there exist $u^{\prime}, l^{\prime} \in \mathrm{F}(L), u^{\prime}$ upper and $l^{\prime}$ lower A-semicontinuous, such that

$$
u \leq u^{\prime} \leq l^{\prime} \leq l \quad \text { and } \quad \iota(u, l)^{\#}=\iota\left(u, u^{\prime}\right)^{\#}=\iota\left(u^{\prime}, l^{\prime}\right)^{\#}=\iota\left(l^{\prime}, l\right)^{\#} .
$$

Theorem 2. Let $L$ be a locale and let $A \subseteq L$ be closed under finite joins with $1 \in A$. Then the following statements are equivalent:

(1) $A$ is $G_{\delta}$-perfect in $L$.

(2) For any upper A-semicontinuous $u \in \mathrm{F}(L)$ and any lower A-semicontinuous $l \in \mathrm{F}(L)$ such that $u \leq l$, there exist $u^{\prime}, l^{\prime} \in \mathrm{F}(L), u^{\prime}$ upper and $l^{\prime}$ lower A-semicontinuous, such that

$$
u \leq u^{\prime} \leq l^{\prime} \leq l \quad \text { and } \quad \iota(u, l)=\iota\left(u, u^{\prime}\right)=\iota\left(u^{\prime}, l^{\prime}\right)=\iota\left(l^{\prime}, l\right)
$$

Theorem 3. Let $L$ be a locale and let $A$ be a sublattice of $L$. Then the following statements are equivalent:

(1) $A$ is perfectly normal in $L$.

(2) For any upper A-semicontinuous $u \in \mathrm{F}(L)$ and any lower A-semicontinuous $l \in \mathrm{F}(L)$ such that $u \leq l$, there exists an $A$-continuous $f \in \mathrm{F}(L)$ such that

$$
u \leq f \leq l \quad \text { and } \quad \iota(u, f)=\iota(f, l)=\iota(u, l) .
$$

The results above have interesting counterparts in the dual case of extremally disconnected locales (in the vein of the approach in [10] and [8]).

Specifically, the dual notion of an $A$ normal in $L$ is the notion of an $A$ extremally disconnected in $L$ ([10]): $A$ is extremally disconnected in $L$ if for any $a, b \in A$ with $a \wedge b=0$ there exist $u, v \in A$ such that $a \wedge u=0=b \wedge v$ and $u \vee v=1$. On the other hand, the dual in $\mathcal{S}(L)$ of the perfectness condition above means that every closed sublocale $\mathfrak{c}(a)$ with $a \in A$ is equal to some $\mathfrak{o}\left(\bigvee_{b \in B}\right)$ for a countable $B \subseteq A$, that is,

every $a \in A$ is complemented in $L$, with complement $\bigvee_{b \in B} b$ for some countable $B \subseteq A$.

When this holds we say that $A$ is countably complemented in $L$ (coincidentally, the dual of the $G_{\delta}$-perfect condition is also equivalent to countable complementedness). 
The reader will have no difficulties then in obtaining the dual of Theorem 3 (since the notions of upper $A$-semicontinuity and lower $A$-semicontinuity are dual to each other [10]):

Corollary 4. Let $L$ be a locale and let $A$ be a sublattice of $L$. Then $A$ is countably complemented and extremally disconnected in $L$ if and only if for any lower A-semicontinuous $u \in \mathrm{F}(L)$ and any upper A-semicontinuous $l \in$ $\mathrm{F}(L)$ such that $u \leq l$, there exists an A-continuous $f \in \mathrm{F}(L)$ such that

$$
u \leq f \leq l \quad \text { and } \quad \iota(u, f)=\iota(f, l)=\iota(u, l) .
$$

Note. In the case of an $A$ closed under countable joins (like the set $\operatorname{Coz} L$ of cozero elements), $A$ is countably complemented in $L$ iff it is complemented in $L$ with complements in $A$, and the latter clearly implies that $A$ is extremally disconnected in $L$. Hence $A$ is countably complemented and extremally disconnected in $L$ iff it is complemented in $L$ (with complements in $A$ ).

\section{References}

[1] Aull, C.E., Thron, W.J.: Separation axioms between $T_{0}$ and $T_{1}$. Indag. Math. 24, 26-37 (1963)

[2] Banaschewski, B.: The real numbers in pointfree topology. Textos de Matemática, vol. 12, University of Coimbra (1997)

[3] Gutiérrez García, J., Kubiak, T.: A new look at some classical theorems on continuous functions on normal spaces. Acta Math. Hung. 119, 333-339 (2008)

[4] Gutiérrez García, J., Kubiak, T.: Inserting measurable functions precisely. Czechoslovak Math. J. 64 (139), 743-749 (2014)

[5] Gutiérrez García, J., Kubiak, T., Picado, J.: Pointfree forms of Dowker's and Michael's insertion theorems. J. Pure Appl. Algebra 213, 98-108 (2009)

[6] Gutiérrez García, J., Kubiak, T., Picado, J.: Localic real functions: A general setting. J. Pure Appl. Algebra 213, 1064-1074 (2009)

[7] Gutiérrez García, J., Kubiak, T., Picado, J.: Perfectness in locales. Quaest. Math. 40, 507-518 (2017)

[8] Gutiérrez García, J., Kubiak, T., Picado, J.: On hereditary properties of extremally disconnected frames and normal frames. Topology Appl. 273, 106978, 15 pp. (2020)

[9] Gutiérrez García, J., Picado, J.: Rings of real functions in pointfree topology. Topology Appl. 158, 2264-2278 (2011)

[10] Gutiérrez García, J., Picado, J.: On the parallel between normality and extremal disconnectedness. J. Pure Appl. Algebra 218, 784-803 (2014)

[11] Johnstone, P.T.: Stone Spaces. Cambridge Univ. Press, Cambridge (1982)

[12] Lane, E.P.: Insertion of a continuous function. Topology Proc. 4, 463-478 (1979)

[13] Li, Y.-M., Wang, G.-J.: Localic Katětov-Tong insertion theorem and localic Tietze extension theorem. Comment. Math. Univ. Carolin. 38, 801-814 (1997)

[14] Mozo Carollo, I.: A lattice-theoretic approach to arbitrary real functions on frames. Quaest. Math. 41, 319-347 (2018) 
[15] Picado, J., Pultr, A.: Frames and Locales: Topology without points. Springer, Basel (2012)

[16] Picado, J., Pultr, A.: A Boolean extension of a frame and a representation of discontinuity. Quaest. Math. 40, 1111-1125 (2017)

[17] Yan, P.-F., Yang, E.-G.: Semi-stratifiable spaces and the insertion of semicontinuous functions. J. Math. Anal. Appl. 328, 429-437 (2007)

Javier Gutiérrez García*

Departamento de Matemáticas

Universidad del País Vasco UPV/EHU

48080 Bilbao

Spain

e-mail: javier.gutierrezgarcia@ehu.eus

Tomasz Kubiak

Wydział Matematyki i Informatyki

Uniwersytet im. Adama Mickiewicza

61-614 Poznań

Poland

e-mail: tkubiak@amu.edu.pl

Jorge Picado

University of Coimbra

CMUC, Department of Mathematics

3001-501 Coimbra

Portugal

e-mail: picado@mat.uc.pt 